\title{
Embedding of the Brane into Six Dimensions
}

\author{
Merab Gogberashvili \\ Andronikashvili Institute of Physics, 6 Tamarashvili Str., Tbilisi 380077, Georgia \\ E-mail: gogber@hotmail.com
}

October 27, 2018

\begin{abstract}
Embedding of the brane metric into Euclidean $(2+4)$-space is found. Brane geometry can be visualized as the surface of the hyper-sphere in six dimensions which 'radius' is governed by the cosmological constant. Minkowski space in this picture is lied on the intersection of this surface with the plane formed by the extra space-like and time-like coordinates.
\end{abstract}

PACS numbers: 04.50.+h, 04.20.Jb, 98.80.Cq

There has been great interest in recent years in models with extended extra dimensions. Ordinary gravity can be recovered if the observable universe is represented by a brane embedded in a higherdimensional space with a non-factorizable geometry [1].

The useful method to study brane models can be the embedding theory [2]. It is well known that $n$-dimensional space-time can be embedded into $N$-dimensional pseudo-Euclidean space with $n \leq N \leq n(n+1) / 2$ [2, 3]. Thus, no more than ten dimensions are required to embed any 4dimensional solution of Einstein's equations with arbitrary energy-momentum tensor. There also exists Campbell's theorem [4], which implies that any solution of $n$-dimensional Einstein's equations can be embedded, at least locally, in a space-time that is itself a solution of $(n+1)$-dimensional, vacuum Einstein's equations [5. Several authors tried to interpreted the embedding as producing an effective stress-energy tensor in low dimensions [6].

The embedding procedure is also interesting from a purely mathematical point of view. It allows invariant classification of known solutions of Einstein's equations to be made [7. Furthermore, the embedding method may lead to new solutions. For example, the maximal analytic extension of Schwarzschild solution was independently found in this way [8].

Embedding of the space-time with the coordinates $x^{\alpha}$ and metric $g_{\alpha \nu}$ into pseudo-Euclidean space with the coordinates $X^{A}$ and with the flat metric $\eta_{A B}$ is given by

$$
d s^{2}=g_{\alpha \nu} d x^{\alpha} d x^{\nu}=\eta_{A B} \partial_{\alpha} X^{A} \partial_{\nu} X^{B} d x^{\alpha} d x^{\nu}=\eta_{A B} d X^{A} d X^{B} .
$$

For example, it is well known [9], that Schwarzschild metric

$$
d s^{2}=\left(1-\frac{2 m}{r}\right) d t^{2}-\frac{d r^{2}}{(1-2 m / r)}-r^{2} d \Omega^{2}
$$

admits isometric embedding of class 2 into Euclidean $(2+4)$-space. Embedding functions in this case are:

$$
\begin{array}{rlrl}
X^{1} & =\sqrt{1-\frac{2 m}{r}} \cos t, & X^{2} & =\sqrt{1-\frac{2 m}{r}} \sin t, \\
X^{4} & =r \sin \theta \cos \varphi, & X^{3}=f(r), \\
X^{5} & =r \sin \theta \sin \varphi, & X^{6}=r \cos \theta,
\end{array}
$$


where $f(r)$ is solution of the equation

$$
f^{\prime 2}=\left(m^{2} / r^{4}+2 m / r\right) /(1-2 m / r) .
$$

In this paper we want to present embedding of the brane metric (which was introduced in [1]) into 6 -dimensional pseudo-Euclidean space with the same signature $(2+4)$ as for the Schwarzschild case. Necessity of two time directions for embedding of $P$ - and $M$-branes was shown in 10 ].

We looking for the functions $X^{A}$ which fulfill the relation

$$
d s^{2}=e^{2 a \xi} d l^{2}-d \xi^{2}=d X_{0}^{2}-d X_{1}^{2}-d X_{2}^{2}-d X_{3}^{2}+d X_{\tau}^{2}-d X_{\kappa}^{2}
$$

Here

$$
l=\sqrt{t^{2}-x^{2}-y^{2}-z^{2}}
$$

is the length in 4-dimensional Minkowski space-time, $\xi$ is the fifth coordinate orthogonal to the brane and $a$ is the parameter connected with the 5 -dimensional cosmological constant $\pm \Lambda$. For the simplicity on the brane we considering Minkowski metric. Ricci tensor of the 5-dimensional space-time where the cosmological constant $\Lambda$ appears is not zero [1], while we assume that bulk (2+4)-space to be pseudoEuclidean again. This can be interpreted as a kind of geometrical introduction of the cosmological constant.

It can be checked that embedding (5) is done by the functions

$$
\begin{array}{r}
X_{\alpha}=e^{a \xi} x_{\alpha}, \\
X_{\tau}=\left(l^{2}-\frac{1}{4}\right) e^{a \xi}-\frac{1}{a^{2}} e^{-a \xi}, \\
X_{\kappa}=\left(l^{2}+\frac{1}{4}\right) e^{a \xi}-\frac{1}{a^{2}} e^{-a \xi},
\end{array}
$$

where $x^{\alpha}$ are coordinates of Minkowski space-time and index $\alpha$ runs over $0,1,2,3$.

Inverse expression of 5 -dimensional coordinates by the embedding functions has the form

$$
\begin{gathered}
x_{\alpha}=\frac{X_{\alpha}}{2\left(X_{\kappa}-X_{\tau}\right)}, \\
\xi=a \ln \left[2\left(X_{\kappa}-X_{\tau}\right)\right] .
\end{gathered}
$$

The geometry of 5-dimensional metric (5) can be visualized as the surface of the hyper-sphere in six dimensions, since

$$
X_{0}^{2}-X_{1}^{2}-X_{2}^{2}-X_{3}^{2}+X_{\tau}^{2}-X_{\kappa}^{2}=\frac{1}{a^{2}} .
$$

The radius of this 'sphere' is $1 /|a|$ and thus governed by the value of the 5 -dimensional cosmological constant $\pm \Lambda$. In this picture 4-dimensional Minkowski space-time is the intersection of this hypersphere with the plane

$$
X_{\kappa}-X_{\tau}=\frac{1}{2}
$$

where $X_{\kappa}$ and $X_{\tau}$ are respectively extra space-like and time-like coordinates of the 6-dimensional space-time.

Possibly here we have correlations with the situation of the linearization of conformal group, the symmetry group of the main equations of physics in zero-mass limit. A long time ago it was discovered that the non-linear 15-parameter conformal transformations can be written as a linear Lorentz-type transformation in a (2+4)-space. For this case the intersection of the null 6-cone with the null 5-plane forming by the extra time-like and space-like coordinates has induced metric of the Minkowski form (for these subjects see, for example, [11]) and formulae of this embedding are similar to (8) - (10) we have in the branes case.

At the end of the paper we want to note that $(2+4)$-space is the interest object for Kaluza-Klein models. For compact extra dimensions this space was studied in [12] and in the context of brane models with non-factorizable geometry in 13 .

Acknowledgements: Author would like to acknowledge the hospitality extended during his visits at the Abdus Salam International Centre for Theoretical Physics where this work was done. 


\section{References}

[1] V. A. Rubakov and M. E. Shaposhnikov, Phys. Lett., B 159, 22 (1985);

M. Gogberashvili, hep-ph/9812296; Mod. Phys. Lett., A14, 2024 (1999); Europhys. Lett., 49, 396 (2000); hep-ph/9908347;

L. Randall and R. Sundrum, Phys. Rev. Lett., 83, 3370 (1999); 83, 4690 (1999).

[2] L. P. Eisenhart, Riemannian Geometry (New Jersey: Princeton University Press, 1949).

[3] A. Friedman, J. Math. Mech., 10, 625 (1961); Rev. Mod. Phys., 37, 201 (1965).

[4] J. E. Campbell, A Course of Differential Geometry (Oxford: Clarendon Press, 1926).

[5] C. Romero, R. Tavakol and R. Zalaletdinov, Gen. Rel. Grav., 28, 365 (1996).

[6] J. Ponce de Leon, Gen. Rel. Grav., 20, 539 (1988);

P. Wesson, H. Liu and P. Lim, Phys. Lett., B 298, 69 (1993);

D.J. Mc Manus, J. Math. Phys., 35, 4889 (1994).

[7] D. Kramer, H. Stephani, M. MacCallum and E. Herlt, Exact Solutions of Einstein's Field Equations (Cambridge: Cambridge University Press, 1980).

[8] C. Fronsdal, Phys. Rev., 116, 778 (1959).

[9] J. Rosen, Rev. Mod. Phys., 37, 204 (1965).

[10] L. Andrianopoli, M. Derix, G. Gibbons, C. Herdeiro, A. Santambrogio and A. Van Proeyen, Class. Quantum Grav., 17, 1875 (2000).

[11] R. Penrose and W. Rindler, Spinors and Space-time (Cambridge: Cambridge University Press, 1986).

[12] M. Pavsic, Nuovo Cimento, B41, 397 (1977);

R. L. Ingraham, Nouvo Cimento, B46, 1 (1978); B46, 16 (1978); B46, 217 (1978); B46, 261 (1978); B47, 157 (1978); B50, 233 (1979); B68, 203 (1982); B68, 218 (1982).

[13] M. Gogberashvili, Phys. Lett., B484, 124 (2000);

M. Gogberashvili and P. Midodashvili, Phys. Lett., B515, 447 (2001); hep-th/0111132. 